\title{
HERIDA PENETRANTE ORBITARIA POR ASTA DE TORO
}

\section{ORBITAL PENETRATING WOUND BY A BULL HORN}

\author{
CAMPOS-MOLLO E ${ }^{1}$, PÉREZ-SANTONJA JJ², SAMPER-GIMÉNEZ J1
}

\section{RESUMEN}

Introducción: Las heridas por asta de toro, comunes en países con afición al toreo y festejos relacionados, representan lesiones graves con un elevado grado de contaminación.

Caso clínico: Varón de 19 años que sufrió traumatismo por asta de toro con herida penetrante en párpado superior izquierdo acompañada de fractura del techo de la órbita y hernia grasa. Se indicó profilaxis antibiótica y antitetánica y se reconstruyó quirúrgicamente la herida. Posteriormente, desarrolló una celulitis orbitaria, que evolucionó satisfactoriamente con tratamiento sistémico en unos pocos días. Discusión: El éxito del tratamiento en este tipo de heridas se fundamenta en una exploración meticulosa, diagnóstico temprano, manejo quirúrgico precoz y profilaxis antibiótica y antitetánica.

Palabras claves: Herida orbitaria, asta de toro, lesión orbitaria, lesión penetrante, órbita.

\begin{abstract}
Introduction: Bull horn injuries are severe lesions with a high risk of bacterial contamination, and are common in countries where people are fond of bullfighting and related spectacles.

Case report: A 19-year-old man was referred with a penetrating wound in the superior left eyelid produced by a bull horn and resulting in a fracture of the orbital roof and a fat hernia. Prophylactic antibiotics and tetanus toxoid were administered and surgical reconstruction of the wound performed. Orbital cellulitis did develop, but this responded to systemic antibiotic therapy within a few days.

Discussion: Successful treatment of this type of lesion requires early diagnosis, meticulous surgical exploration and appropriate use of prophylactic antibiotics and tetanus toxoid (Arch Soc Esp Oftalmol 2007; 82: 645-648).
\end{abstract}

Key words: Orbital wound, bull horn, orbit injury, penetrating injury, orbit.

\section{INTRODUCCIÓN}

Las heridas por asta de toro hacen referencia a todas las lesiones que se derivan del encontronazo con los cuernos del animal astado.
Se clasifican siguiendo la terminología taurina en (1-3): a) Varetazo: contusión producida por el choque transversal de la pala del cuerno; b) Puntazo: solución de continuidad de la piel y del tejido celular subcutáneo producida por la punta del asta sin

\footnotetext{
Recibido: 8/9/06. Aceptado: 20/9/07.

Servicio de Oftalmología. Hospital Virgen de los Lirios. Alcoy (Alicante). España.

1 Licenciado en Medicina.

2 Doctor en Medicina.

Correspondencia:

Ezequiel Campos Mollo

Ptda. Alzabares Alto, 145

03290 Elche (Alicante)

España

E-mail: ezechiel@hotmail.com
} 
afectar a planos musculares; c) Cornada: herida incisocontusa con orificio de entrada pequeño que afecta planos musculares o cavidades orgánicas; d) Cornada despistante: herida con orificio de entrada alejado del foco traumático más importante; e) Cornada envainada: lesión de planos profundos con graves lesiones internas sin solución de continuidad de la piel.

La mayoría de estas lesiones (63\%) afectan a miembros inferiores, mientras que las producidas en la cara y cuello comprenden únicamente el 2-3\% $(1-2,4)$.

El total de publicaciones en relación a este tipo de lesiones es muy reducido y en la literatura científica no se han encontrado referencias a heridas orbitarias producidas por este mecanismo.

\section{CASO CLÍNICO}

Un varón de 19 años, empitonado en la suelta de vaquillas de unas fiestas populares, fue remitido a nuestro Servicio desde otro centro asistencial con traumatismo orbitario izquierdo por asta de toro de diez horas de evolución.

La exploración inicial mostró una herida incisa en reborde orbitario superior (puntazo) suturada en el hospital de origen y una herida penetrante (cornada) de bordes anfractuosos en párpado superior izquierdo con hernia de la grasa orbitaria. El diámetro mayor del orificio de entrada midió $1,7 \mathrm{~cm}$ y aproximadamente $1,5 \mathrm{~cm}$ de profundidad desde el reborde orbitario.

Se procedió a una exploración minuciosa de la herida y se comprobó la integridad del globo ocular con tonometría y oftalmoscopía normales. Se lavó exhaustivamente la herida con povidona iodada diluida en solución salina en relación 1:2. Se realizó el desbridamiento del tejido graso herniado y cierre primario de la herida.

La tomografía computarizada (TC) mostró una fractura de techo orbitario izquierdo (fig. 1). Se indicó una dosis de recuerdo de toxoide difteriatétanos y antibioterapia intravenosa de amplio espectro con ciprofloxacino $400 \mathrm{mg}$ cada 12 horas y clindamicina $600 \mathrm{mg}$ cada 8 horas. Cuatro días después del traumatismo, el paciente desarrolló tumefacción palpebral con exudación de la herida, limitación de la supraducción y desplazamiento inferior de globo ocular (figs. 2 y 3 ).

Independientemente de que el origen fuera infeccioso o inflamatorio se tomaron muestras para cul-

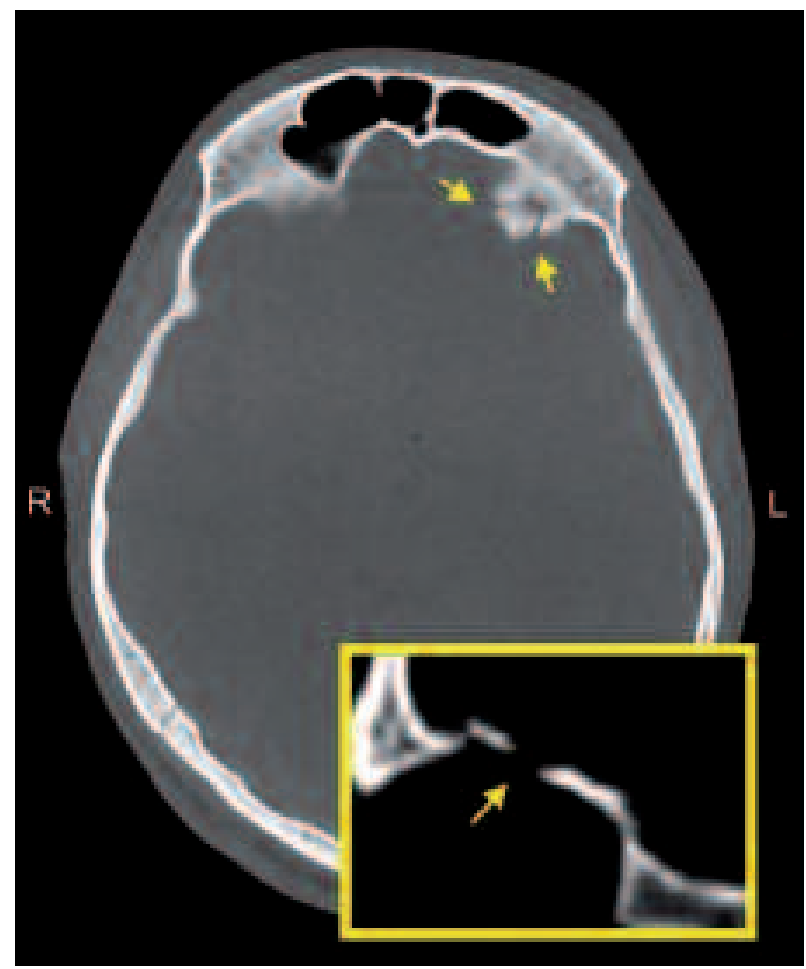

Fig. 1: En el corte axial de la tomografía computarizada aplicando un filtro de hueso se observan las líneas de fractura en el techo orbitario izquierdo. En el corte sagital (construido a partir de cortes axiales) se objetiva el levantamiento de un fragmento óseo.

tivo de los exudados del margen de la herida, se añadió vancomicina 1 gramo IV cada 12 horas para cubrir al Staphylococcus aureus meticilinresistente y se sustituyó la clindamicina por metronidazol 1 gramo cada 8 horas intravenoso, debido a que atraviesa mejor la barrera hematoencefálica cubriendo

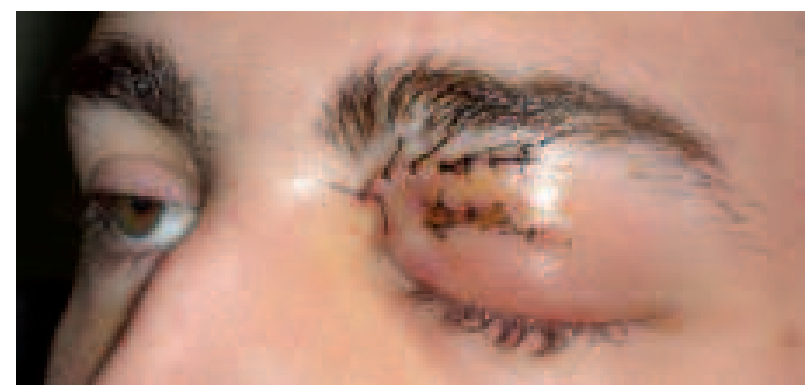

Fig. 2: El paciente desarrolló una celulitis orbitaria. Se puede apreciar una herida incisa o puntazo bajo reborde orbitario (suturada en el hospital de origen) y una herida palpebral de bordes anfractuosos que correspondería con el orificio de entrada de la cornada. 


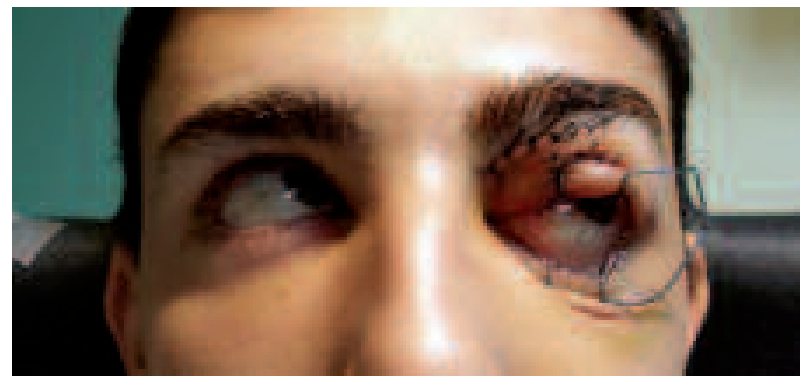

Fig. 3: Facilitando la apertura palpebral con un blefarostato se puede observar un desplazamiento inferior del globo ocular con limitación de la supraducción y la hiperemia conjuntival.

el riesgo potencial de la extensión intracraneal de una infección a través de la fractura del techo orbitario. Además se administraron corticoides sistémicos en pauta descendente.

En el cultivo realizado crecieron colonias de Staphylococcus epidermidis sensible a casi todos los antibióticos testados en el antibiograma constituyendo, probablemente, un agente contaminante de la muestra.

La TC reveló un engrosamiento del músculo recto superior y de partes blandas en relación al proceso inflamatorio con desplazamiento del globo ocular (fig. 4).

El cuadro evolucionó satisfactoriamente, presentando 2 meses después, una agudeza visual sin corrección de unidad, motilidad ocular extrínseca y palpebral normal y una tensión ocular de 12 mm Hg (fig. 5).

\section{DISCUSIÓN}

La vaquilla, por muy pequeña que sea, desarrolla un mecanismo de altísima energía, «la embestida», que es transmitido a sus astas a través de un juego de palancas desde el tercio posterior del animal con un trayecto ascendente $(2,4)$. Además, el animal astado intenta rechazar al oponente mediante la rotación de su cuello originando afectación de múltiples tejidos (2).

Lesiones graves pueden parecer poco evidentes debido a la apariencia externa. Por tanto, el herido por asta de toro debe ser considerado un politraumatizado siendo una indicación prioritaria la realización de técnicas de imagen (radiografía, ecografía, TAC, RMN).

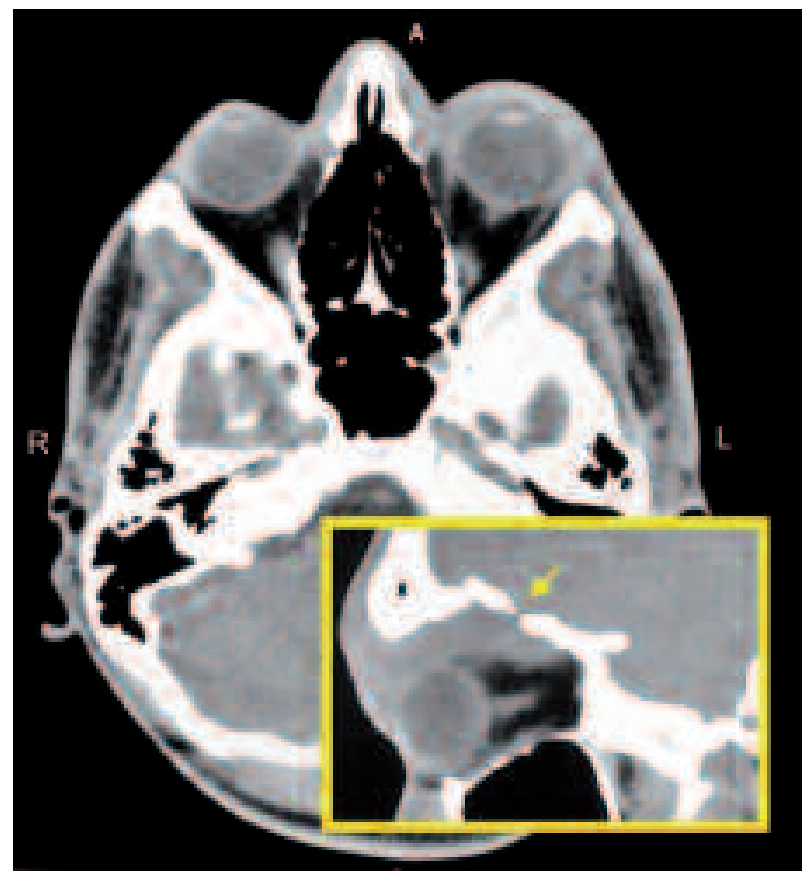

Fig. 4: El corte axial de la tomografía computarizada muestra una tumefacción de partes blandas y en el corte sagital (construido a partir de cortes axiales) se observa la fractura, la inflamación de partes blandas y el desplazamiento inferior del globo ocular.

Es necesario un lavado con solución aséptica, el desbridamiento de la herida y una exploración minuciosa (2). Al final de la intervención, pertenece al cirujano determinar si deja la herida abierta o realiza un cierre primario con correcta adecuación de los bordes y sutura interrumpida siendo obligatoria la colocación de un drenaje (1) en planos ana-

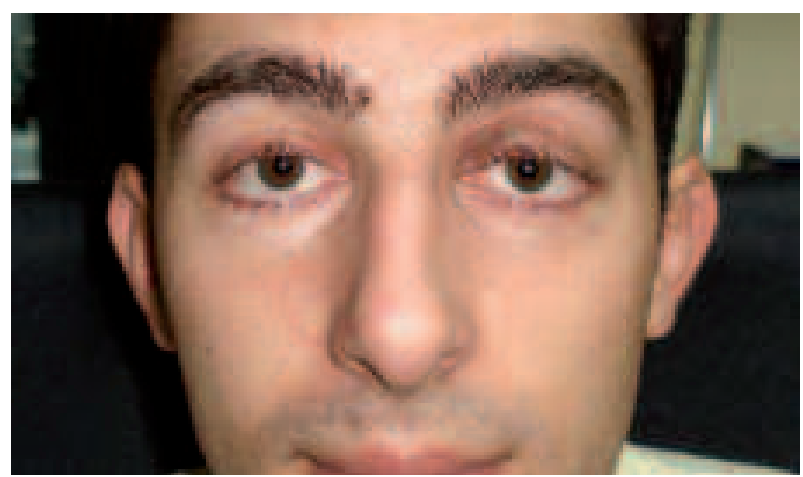

Fig. 5: Resultado dos meses después del trauma taurino. 
tómicos profundos siempre que se pueda garantizar la estabilidad del mismo.

Las heridas por asta de toro son altamente contaminadas debido a la presencia de gérmenes en el asta y el entorno natural por lo que es imperativo completar la profilaxis antitetánica y usar antibióticos de amplio espectro a dosis terapéuticas (12,4).

Podemos concluir que el éxito del tratamiento en este tipo de heridas se fundamenta en un diagnóstico temprano, manejo quirúrgico correcto en el menor tiempo posible desde que se produce el trauma taurino y la profilaxis antibiótica y antitetánica.

\section{BIBLIOGRAFÍA}

1. Hernández E, Gómez-Perlado B, Villaverde M, Vaquero $G$, Marugán JA, Besharat F, García E, del Amo E, Prado A. Heridas por asta de toro. Estudio de 96 pacientes. Cir Esp 1995; 59: 156-159.

2. Mansilla-Roselló A, Fuentes-Martos R, Astruc-Hoffman A, Flores-Arcas A, Albert-Vila A, Fernández-Valdearenas $R$ et al. Estudio de 44 heridas por asta de toro. Cir Esp 1997; 63: 36-39.

3. Zumárraga-Navas P, Sellés-Dechent R, Pardo-Correcher $J M$, Asencio-Arana F, Ruiz del Castillo J. Herida por asta de toro: cornada envainada. Cir Esp 1999; 65: 447-448.

4. Martínez-Ramos D, Miralles-Tena JM, Escrig-Sos J, Traver-Martínez G, Cisneros-Reig I, Salvador-Sanchís JL. Heridas por asta de toro en el Hospital General de Castellón. Estudio de 387 pacientes. Cir Esp 2006; 80: 16-22. 